\title{
A CLASS OF NONLINEAR EVOLUTION EQUATIONS IN A BANACH SPACE
}

\author{
BY \\ J. R. DORROH
}

We treat the nonlinear evolution equation

$$
f^{\prime}(t)=A(t, f(t)) f(t)
$$

where the unknown function $f$ is from a real number interval into a Banach space $X$. For suitable real numbers $t$ and vectors $x$ in $X, A(t, x)$ is the infinitesimal generator of a holomorphic semigroup of linear contraction operators in $X$, and certain regularity requirements are placed on the function $(t, x) \rightarrow A(t, x)$.

After proving a local existence, uniqueness, and stability theorem for $\left({ }^{*}\right)$, we consider the case $A(t, x)=H(x)$ and obtain conditions under which there is a strongly continuous semigroup of nonlinear nonexpansive transformations whose infinitesimal generator is an extension of the transformation $Q x=H(x) x$.

We state our main results in $\S 1$ and prove them in $\$ 2$. In $\$ 3$, we prove some theorems about linear semigroups in a function space which yield examples of our main results and are of some interest in themselves.

1. The main results. Let $X$ be a complex Banach space. If $0<\phi \leqq \pi / 2$, then let $S_{\phi}=\{z \in C: z=0$ or $|\arg z| \leqq \phi\}$, where $C$ denotes the complex plane. Following [6], we denote by $\mathrm{CH}(\phi)$ the collection of all semigroups $\left\{T(z): z \in S_{\phi}\right\}$ of linear contraction operators in $X$ which are holomorphic on int $\left(S_{\phi}\right)$ and strongly continuous on $S_{\phi}$. We denote by GH $(\phi)$ the collection of all infinitesimal generators of semigroups in $\mathrm{CH}(\phi)$.

Let $[a, b]$ be a closed real number interval, $0<\phi \leqq \pi / 2$, and $S$ a closed set in $X$. Let $A$ be a function from $[a, b] \times S \rightarrow \mathrm{GH}(\phi)$ such that the following conditions are satisfied:

$\left(C_{1}\right)$ The operators $A(t, x)$ all have the same domain $D_{0}$.

$\left(\mathrm{C}_{2}\right)$ There is a locally bounded nonnegative function $K$ on $[a, b] \times S$ such that

$$
\left\|[I-A(t, y)][I-A(s, x)]^{-1}-I\right\| \leqq K(s, x)(|s-t|+\|x-y\|)
$$

for $a \leqq s, t \leqq b$ and $x, y \in S$, where $I$ denotes the identity transformation on $X$,

$\left(\mathrm{C}_{3}\right)(\exp [\xi A(t, x)]) S \subset S$ for $\xi \geqq 0, a \leqq t \leqq b$, and $x \in S$, where

$$
\left\{\exp [z A(t, x)] ; z \in S_{\phi}\right\}
$$

is the class $\mathrm{CH}(\phi)$ semigroup generated by $A(t, x)$.

Received by the editors April 1, 1969 and, in revised form, June 9, 1969.

Copyright (C) 1970, American Mathematical Society 
In connection with the condition $\left(\mathrm{C}_{2}\right)$, we mention that the invertibility of $I-A(s, x)$ follows from the fact that $A(s, x) \in \mathrm{GH}(\phi)$, see [6], or [2], which serves also as a general reference for semigroups of operators. The fact that $[I-A(t, y)]$ $\cdot[I-A(s, x)]^{-1}$ is bounded follows from $\left(\mathrm{C}_{1}\right)$ and [3, Lemma 2, p. 212].

Theorem 1. Suppose $x_{0} \in D_{0} \cap S$ and $a \leqq t_{0}<b$. Then there is a number $c$ in $\left(t_{0}, b\right]$ such that there is a unique continuously differentiable function $f$ from $\left[t_{0}, c\right]$ into $D_{0} \cap S$ satisfying $f\left(t_{0}\right)=x_{0}$ and

$$
f^{\prime}(t)=A(t, f(t)) f(t) \text { for } t_{0} \leqq t \leqq c .
$$

Also, if $\varepsilon>0$, then there exists $\delta>0$ such that if $x_{1} \in D_{0} \cap S, t_{0}<c_{1} \leqq c,\left\|x_{0}-x_{1}\right\|$ $<\delta$, and $g$ is a continuously differentiable function from $\left[t_{0}, c_{1}\right]$ into $D_{0} \cap S$ such that $g\left(t_{0}\right)=x_{1}$ and $g^{\prime}(t)=A(t, g(t)) g(t)$ for $t_{0} \leqq t \leqq c_{1}$, then $\|g(t)-f(t)\|<\varepsilon$ for $t_{0} \leqq t \leqq c_{1}$.

Definition 1.1. A semi-inner product on $X$ means a function $[\cdot, \cdot]$ from $X \times X$ into $C$ such that for each $y \in Y,[\cdot, y]$ is a bounded liner functional of norm $\|y\|$, and $[y, y]=\|y\|^{2}$ (see [5]).

DefinITION 1.2. If $[\cdot, \cdot]$ is a semi-inner product on $X$, then a transformation $W$ with domain and range contained in $X$ is said to be dissipative (with respect to $[\cdot, \cdot])$ if $\operatorname{Re}[W x-W y, x-y] \leqq 0$ for $x, y \in D(W)$, the domain of $W$.

REMARK. Throughout this section, $[\cdot, \cdot]$ will denote a fixed semi-inner product on $X$, and all results will be independent of the particular semi-inner product used.

THEOREM 2. Suppose $H$ is a function from $S$ into $\mathrm{GH}(\phi)$ which satisfies conditions $\left(\mathrm{C}_{1}\right),\left(\mathrm{C}_{2}\right)$, and $\left(\mathrm{C}_{3}\right)$; more precisely, the function $(t, x) \rightarrow H(x)$ satisfies these conditions. Suppose $D_{0} \cap S$ is dense in $S$ and define $Q$ on $D_{0} \cap S$ by $Q x=H(x) x$. Suppose $Q$ is dissipative. Then there is a unique strongly continuous semigroup $\{T(t) ; t \geqq 0\}$ of nonexpansive nonlinear transformations from $S$ into $S$ such that for each $x$ in $D_{0} \cap S, T(\cdot) x$ is a continuously differentiable function from $[0, \infty)$ into $D_{0} \cap S$, and $(d / d t) T(t) x=Q T(t) x$ for $t \geqq 0$.

2. Proof of the main theorems. We will call a function $B$ from a number interval $[0, R]$ into $\mathrm{GH}(\phi)$ regular if the following conditions are satisfied:

$\left(\mathrm{R}_{1}\right) B(t)$ has domain $D_{0}$ for $0 \leqq t \leqq R$.

$\left(\mathrm{R}_{2}\right)$ There is a positive constant $L$ such that

$$
\left\|[I-B(t)][I-B(s)]^{-1}-I\right\| \leqq L|t-s|
$$

for $0 \leqq s, t \leqq R$.

$\left(\mathrm{R}_{3}\right)(\exp [\xi B(t)]) S \subset S$ for $\xi \geqq 0$ and $0 \leqq t \leqq R$.

We point out that a regular operator function $B$ on $[0, R]$ also satisfies:

$\left(\mathrm{R}_{4}\right)\left\|[I-B(t)][I-B(s)]^{-1}\right\| \leqq 1+L R$ for $0 \leqq s, t \leqq R$, where $L$ is as in $\left(\mathrm{R}_{2}\right)$.

$$
\begin{aligned}
&\left\|[I-B(r)][I-B(s)]^{-1}-[I-B(t)][I-B(s)]^{-1}\right\| \\
& \leqq\left\|[I-B(r)][I-B(t)]^{-1}-I\right\| \cdot\left\|[I-B(t)][I-B(s)]^{-1}\right\| \\
& \leqq|r-t|(1+L R) L .
\end{aligned}
$$


LemMA 2.1. Suppose $B$ is a regular operator function on $[0, R]$, and $\beta$ is a positive nonincreasing function on $[0, R]$ with Lipschitz constant $L^{\prime}$. Define the operator function $A$ on $[0, R]$ by $A(t)=\beta(t)[B(t)-I]$.

Then $A$ satisfies Tanabe's conditions $1^{0}$ and $2^{\circ}$ of [8]. In particular, let $0<\phi_{1}<\phi$ and define

Also define

$$
\Sigma=\left\{\lambda \in C: \lambda=0 \text { or }|\arg \lambda| \leqq \phi_{1}+\pi / 2\right\}
$$

$$
\begin{aligned}
M & =\left[\beta(R) \sin \left(\phi-\phi_{1}\right)\right]^{-1}\left[\left(1-\sin \phi_{1}\right) / 2\right]^{-1 / 2}, \\
K & =\left(L \beta(0)+L^{\prime}\right)(1+L R) / \beta(R),
\end{aligned}
$$

where $L$ is as in $\left(\mathrm{R}_{2}\right)$. Then $A$ satisfies the conditions:

( $\left.\mathrm{T}_{1}\right) \rho(A(t)) \supset \Sigma$ for $0 \leqq t \leqq R$, and

$$
\left\|[\lambda I-A(t)]^{-1}\right\| \leqq M /(|\lambda|+1)
$$

for $\lambda \in \Sigma$ and $0 \leqq t \leqq R$. (If $T$ is an operator in $X$, then $\rho(T)$ denotes the resolvent set of $T$.)

$\left(\mathrm{T}_{2}\right)\left\|A(r) A(s)^{-1}-A(t) A(s)^{-1}\right\| \leqq K|r-t|$ for $0 \leqq r, s, t \leqq R$.

Proof. Let $0 \leqq s, t, r \leqq R$. Define

$$
\Delta_{\phi}=\{\lambda \in C: \lambda=0 \text { or }|\arg \lambda| \geqq \phi+\pi / 2\} .
$$

Then $\beta(t) B(t) \in \mathrm{GH}(\phi)$, and $\Sigma \subset C \backslash \Delta_{\phi}$, so $\rho(\beta(t) B(t)) \supset \Sigma$ and $\left\|[\lambda I-\beta(t) B(t)]^{-1}\right\|$ $\leqq 1 / d\left(\lambda, \Delta_{\phi}\right)$ for $\lambda \in \Sigma$, see [6]. Also $\lambda I-A(t)=[\lambda+\beta(t)] I-\beta(t) B(t)$, and $\lambda+\beta(t) \in \Sigma$ if $\lambda \in \Sigma$, so $\|\left[\lambda I-A(t)^{-1} \| \leqq 1 / d\left(\lambda+\beta(t), \Delta_{\phi}\right)\right.$ for $\lambda \in \Sigma$. Property $\left(\mathrm{T}_{1}\right)$ follows from this and the fact that $d\left(\lambda+\beta(t), \Delta_{\phi}\right) \geqq(|\lambda|+1) / M$ for $\lambda \in \Sigma$.

Let $A_{\xi}=A(\xi), B_{\xi}=B(\xi)$, and $\beta_{\xi}=\beta(\xi)$ for $0 \leqq \xi \leqq R$. Then

$$
\begin{aligned}
A_{r} A_{s}^{-1}-A_{t} A_{s}^{-1}= & \beta_{s}^{-1} \beta_{r}\left[\left(I-B_{r}\right)\left(I-B_{s}\right)^{-1}-\left(I-B_{t}\right)\left(I-B_{s}\right)^{-1}\right] \\
& +\beta_{s}^{-1}\left(\beta_{r}-\beta_{t}\right)\left(I-B_{t}\right)\left(I-B_{s}\right)^{-1},
\end{aligned}
$$

so that $\left(\mathrm{T}_{2}\right)$ follows from $\left(\mathrm{R}_{4}\right)$ and $\left(\mathrm{R}_{5}\right)$.

LEMMA 2.2. Let $F$ be a function from $[0, R]$ into $[a, b]$ with Lipschitz constant $L_{1}$, and $\psi$ a function from $[0, R]$ into $S$ with Lipschitz constant $L_{2}$. Define the operator function $B$ on $[0, R]$ by

$$
B(t)=A(F(t), \psi(t)) .
$$

Then $B$ is regular, where we can take the constant $L$ of $\left(\mathrm{R}_{2}\right)$ as

see $\left(\mathrm{C}_{2}\right)$.

$$
L=\left(L_{1}+L_{2}\right) \sup _{0 \leqq t \leqq R} K(F(t), \psi(t))
$$

Thus if $\beta$ is positive, nonincreasing, and Lipschitz continuous on $[0, R]$, and we define $A(t)=\beta(t)[B(t)-I]$ for $0 \leqq t \leqq R$, then $A$ satisfies $\left(\mathrm{T}_{1}\right)$ and $\left(\mathrm{T}_{2}\right)$.

If in addition, $\beta(t)-\beta(s) \leqq-(t-s) \beta(0) L$ for $0 \leqq s \leqq t \leqq R$, then $A$ also satisfies

$$
\left\|A(t) A(s)^{-1}\right\| \leqq 1 \text { for } 0 \leqq s \leqq t \leqq R .
$$

Proof. Only the last statement needs proof, and it follows from the fact that $\beta(t) \beta(s)^{-1} \leqq e^{[\beta(t)-\beta(s)] / \beta(s)}$, and $\left\|(I-B(t))(I-B(s))^{-1}\right\| \leqq e^{L(t-s)}$ for $0 \leqq s \leqq t \leqq R$. 
Lemma 2.3. Let the operator function $A:[0, R] \rightarrow \mathrm{GH}(\phi)$ be as in Lemma 2.1, and let $x_{0} \in D_{0}$. Then there is a unique continuously differentiable function $f$ from $[0, R]$ into $D_{0}$ such that $f(0)=x_{0}$ and $f^{\prime}(t)=A(t) f(t)$ for $0 \leqq t \leqq R$.

Proof. Tanabe establishes much more than this in [8].

LEMMA 2.4. Let $A, x_{0}$, and $f$ be as in Lemma 2.3. If $\Delta=\left\{t_{0}, \ldots, t_{n}\right\}$ is a partition of $[0, R]$, then let $f_{\Delta}$ be defined on $[0, R]$ by $f_{\Delta}(0)=x_{0}$, and

$$
f_{\Delta}(t)=T_{k}\left(t-t_{k-1}\right) f_{\Delta}\left(t_{k-1}\right) \text { for } t_{k-1} \leqq t \leqq t_{k},
$$

where $T_{k}(\xi)=\exp \left[\xi A\left(t_{k}\right)\right]$. Then $f_{\Delta}$ converges uniformly to $f$ on $[0, R]$ as the norm of $\Delta$ approaches zero.

Proof. Define $A_{\Delta}$ on $[0, R]$ by $A_{\Delta}(0)=A\left(t_{1}\right)$ and $A_{\Delta}(t)=A\left(t_{k}\right)$ for $t_{k-1}<t \leqq t_{k}$. Then $f_{\Delta}^{\prime}(t)=A_{\Delta}(t) f_{\Delta}(t)$ for $t \in[0, R] \backslash \Delta$.

Let $h_{\Delta}(t)=f(t)-f_{\Delta}(t)$ for $0 \leqq t \leqq R$. Then

$$
\begin{aligned}
h_{\Delta}^{\prime}(t) & =\left[A(t)-A_{\Delta}(t)\right] f(t)+A_{\Delta}(t) h_{\Delta}(t) \\
& =\left[I-A_{\Delta}(t) A(t)^{-1}\right] f^{\prime}(t)+A_{\Delta}(t) h_{\Delta}(t) .
\end{aligned}
$$

By [4, Lemma 1.3, p. 510], $\left\|h_{\Delta}(t)\right\|(d / d t)\left\|h_{\Delta}(t)\right\|=\operatorname{Re}\left[h_{\Delta}^{\prime}(t), h_{\Delta}(t)\right]$ a.e. on $[0, R]$. Thus $(d / d t)\left\|h_{\Delta}(t)\right\| \leqq K|\Delta| \Lambda$ a.e. on $[0, R]$, where $K$ is as in $\left(\mathrm{T}_{2}\right)$,

$$
\Lambda=\sup _{0 \leqq t \leqq R}\left\|f^{\prime}(t)\right\|
$$

and $|\Delta|$ denotes the norm of $\Delta$. We have used the fact that $A_{\Delta}(t)$ is dissipative, see [5].

Lemma 2.5. Let $A, x_{0}$, and $f$ be as in Lemma 2.3. Then

$$
\|f(t)\| \leqq\|f(0)\| \exp \left[-\int_{0}^{t} \beta\right]
$$

for $0 \leqq t \leqq R$.

If $A$ satisfies $\left(\mathrm{T}_{3}\right)$, then $\left\|f^{\prime}(t)\right\| \leqq\left\|f^{\prime}(0)\right\| \exp \left[-\int_{0}^{t} \beta\right]$ for $0 \leqq t \leqq R$.

Proof. Let $\Delta, f_{\Delta}$, and $T_{k}$ be as in Lemma 2.4. Then $T_{k}(\xi)=e^{-\xi \beta\left(t_{k}\right)} \exp \left[\xi \beta\left(t_{k}\right) B\left(t_{k}\right)\right]$, so that $\left\|T_{k}(\xi)\right\| \leqq e^{-\xi \beta\left(t_{k}\right)}$. Therefore,

$$
\left\|f_{\Delta}(t)\right\| \leqq\|f(0)\| \exp \left[-\beta\left(t_{k}\right)\left(t-t_{k-1}\right)-\sum_{j=1}^{k-1} \beta\left(t_{j}\right)\left(t_{j}-t_{j-1}\right)\right]
$$

for $t_{k-1} \leqq t \leqq t_{k}$, and the first conclusion follows.

Define $X_{k}=T_{k}\left(t_{k}-t_{k-1}\right), A_{k}=A\left(t_{k}\right)$, and $\beta_{k}=\beta\left(t_{k}\right)$ for $k=0,1, \ldots, n$.

If $t_{k-1}<t<t_{k}$, then

$$
\begin{aligned}
f_{\Delta}^{\prime}(t) & =A_{k} f_{\Delta}(t) \\
& =A_{k} T_{k}\left(t-t_{k-1}\right) X_{k-1} \cdots X_{1} \\
& =T_{k}\left(t-t_{k-1}\right) A_{k} A_{k-1}^{-1} A_{k-1} X_{k-1} \cdots X_{1} \\
& =T_{k}\left(t-t_{k-1}\right) A_{k} A_{k-1}^{-1} X_{k-1} \cdots X_{1} A_{1} A_{0}^{-1} A_{0} x_{0}
\end{aligned}
$$

and the second conclusion follows. 
Lemma 2.6. Let $A, x_{0}$, and $f$ be as in Lemma 2.3, but add the condition that $x_{0} \in S$. Then $\left(\exp \left[\int_{0}^{t} \beta\right]\right) f(t) \in S$ for $0 \leqq t \leqq R$.

Proof. Let $\Delta$ and $f_{\Delta}$ be as in Lemma 2.4. From $\left(\mathbf{R}_{3}\right)$ and the construction of $f_{\Delta}$, we get

$$
\left(\exp \left[\left(t-t_{k-1}\right) \beta\left(t_{k-1}\right)+\sum_{j=1}^{k-1} \beta\left(t_{j}\right)\left(t_{j}-t_{j-1}\right)\right]\right) f_{\Delta}(t) \in S
$$

for $t_{k-1} \leqq t \leqq t_{k}$.

2.7. Proof of Theorem 1. Choose $\delta>0$ so that $K(t, x)$ (see condition $\left(\mathrm{C}_{2}\right)$ ) is bounded for $\left|t-t_{0}\right| \leqq \delta,\left\|x-x_{0}\right\| \leqq \delta$. Let $K_{0}$ be an upper bound for $K(t, x)$ on this set, with $K_{0}>1,(1 / \delta)$. Let

$$
\begin{aligned}
y_{0} & =A\left(t_{0}, x_{0}\right) x_{0}, & \gamma & =2 K_{0}\left(1+2\left\|x_{0}\right\|+\left\|y_{0}\right\|\right), \\
c & =\min \left[b, t_{0}+(1 / 2 \gamma)\right], & R & =-\gamma^{-1} \ln \left(1-\gamma\left(c-t_{0}\right)\right) .
\end{aligned}
$$

We will need the following two inequalities, which follow immediately from the above definitions:

$$
\begin{gathered}
R\left(2\left\|x_{0}\right\|+\left\|y_{0}\right\|\right) \leqq \delta, \\
c-t_{0} \leqq \delta .
\end{gathered}
$$

Define $F$ from $[0, R]$ onto $\left[t_{0}, c\right]$ by $F(\tau)=t_{0}+\gamma^{-1}\left[1-e^{-\gamma \tau}\right]$. Define $\beta$ on $[0, R]$ by $\beta(\tau)=e^{-\gamma \tau}$, and define $G$ from $\left[t_{0}, c\right]$ onto $[0, R]$ by $G(t)=-\gamma^{-1} \ln \left[1-\gamma\left(t-t_{0}\right)\right]$. Then

$$
\begin{gathered}
F(G(t))=t, \quad G(F(\tau))=\tau, \\
G^{\prime}(t) \beta(G(t))=1, \\
\int_{0}^{\tau} \beta=F(\tau)-t_{0} .
\end{gathered}
$$

Define $\alpha$ on $[0, R]$ by $\alpha(\tau)=\exp \left(\int_{0}^{\tau} \beta\right)$.

We intend to solve $(*)$ by first solving

$$
g^{\prime}(\tau)=\beta(\tau)[A(F(\tau), \alpha(\tau) g(\tau))-I] g(\tau),
$$

and then making the substitution $f(t)=e^{t-t_{0}} g(G(t))$. (2.7.3), (2.7.4), and (2.7.5) are the pertinent identities for showing that this yields a solution of $\left(^{*}\right)$.

We define inductively the sequence $\left\{g_{n}\right\}$ of functions on $[0, R]$ as follows:

where

$$
g_{0}(\tau)=x_{0}, \quad g_{n+1}(0)=x_{0}, \quad g_{n+1}^{\prime}(\tau)=A_{n}(\tau) g_{n+1}(\tau)
$$

$$
A_{n}(\tau)=\beta(\tau)\left[A\left(F(\tau), \psi_{n}(\tau)\right)-I\right], \quad \psi_{n}(\tau)=\alpha(\tau) g_{n}(\tau)
$$

We see that this inductive definition is possible by Lemmas 2.2, 2.3, and 2.6. 
We will need the fact that each of the operator functions $A_{n}$ has property $\left(\mathrm{T}_{3}\right)$; in fact this is the reason for our change of variable. Define $B_{n}(\tau)=A\left(F(\tau), \psi_{n}(\tau)\right)$ for $0 \leqq \tau \leqq R$, and $n=0,1,2,3, \ldots$ Then each $B_{n}$ is regular by Lemma 2.2. For each $n$, let $L^{(n)}$ denote the least constant $L$ that will work in condition $\left(\mathrm{R}_{2}\right)$ for $B_{n}$. Notice that

$$
[\beta(\tau)-\beta(\sigma)] / \beta(0) \leqq-\gamma B(R)(\tau-\sigma) \leqq-(\gamma / 2)(\tau-\sigma)
$$

for $0 \leqq \sigma \leqq \tau \leqq R$. Thus by Lemma $2.2, A_{n}$ satisfies $\left(\mathrm{T}_{3}\right)$ if $L^{(n)} \leqq(\gamma / 2)$. In order to show this we will need

$$
\begin{aligned}
\left|F^{\prime}(\tau)\right|= & \left|e^{-\gamma \tau}\right| \leqq 1 \\
\left|\alpha^{\prime}(\tau)\right|= & \left|\exp \left[-\gamma \tau+\int_{0}^{\tau} \beta\right]\right| \leqq 1, \\
& \left|F(\tau)-t_{0}\right| \leqq \delta
\end{aligned}
$$

Thus, we have $L_{0} \leqq K_{0}\left(1+\left\|x_{0}\right\|\right)<\gamma / 2$ since $\left\|\psi_{0}(\tau)-x_{0}\right\|<R\left\|x_{0}\right\| \leqq \delta,\left\|\psi_{0}^{\prime}(\tau)\right\|$ $\leqq\left\|x_{0}\right\|$, so that $A_{0}$ has property $\left(\mathrm{T}_{3}\right)$.

Suppose $A_{n}$ has property $\left(\mathrm{T}_{3}\right)$. Then

$$
\begin{gathered}
\psi_{n+1}^{\prime}(\tau)=\alpha(\tau) g_{n}^{\prime}(\tau)+\alpha^{\prime}(\tau) g_{n}(\tau), \\
\left\|\psi_{n+1}^{\prime}(\tau)\right\| \leqq\left\|g_{n}^{\prime}(0)\right\|+\left\|x_{0}\right\| \leqq 2\left\|x_{0}\right\|+\left\|y_{0}\right\|
\end{gathered}
$$

by Lemma 2.5 , and (2.7.7). Therefore,

$$
\left\|\psi_{n+1}(\tau)-x_{0}\right\| \leqq \delta
$$

by (2.7.1). Thus $L^{(n+1)} \leqq K_{0}\left(1+2\left\|x_{0}\right\|+\left\|y_{0}\right\|\right)=\gamma / 2$ by (2.7.6), (2.7.8), and Lemma 2.2. Thus $A_{n+1}$ also has property $\left(\mathrm{T}_{3}\right)$.

Thus, we have

$$
\left\|g_{n}^{\prime}(\tau)\right\| \leqq\left(\left\|y_{0}\right\|+\left\|x_{0}\right\|\right) / \alpha(\tau)
$$

for $0<\tau \leqq R$, and $n=0,1,2, \ldots$ by Lemma 2.5 .

For each $n=1,2,3, \ldots$, define $h_{n}$ on $[0, R]$ by $h_{n}(\tau)=g_{n+1}(\tau)-g_{n}(\tau)$. Then

$$
\begin{aligned}
h_{n}^{\prime}(\tau) & =A_{n}(\tau) g_{n+1}(\tau)-A_{n-1}(\tau) g_{n}(\tau) \\
& =\left[A_{n}(\tau)-A_{n-1}(\tau)\right] g_{n+1}(\tau)+A_{n-1}(\tau) h_{n}(\tau) \\
& =\left[I-A_{n-1}(\tau) A_{n}(\tau)^{-1}\right] g_{n+1}^{\prime}(\tau)+A_{n-1}(\tau) h_{n}(\tau) .
\end{aligned}
$$

By $[4$, Lemma 1.3 , p. 510$]$, we have

$$
\left\|h_{n}(\tau)\right\|(d / d \tau)\left\|h_{n}(\tau)\right\|=\operatorname{Re}\left[h_{n}^{\prime}(\tau), h_{n}(\tau)\right]
$$

a.e. on $[0, R]$, so that $(d / d \tau)\left\|h_{n}(\tau)\right\| \leqq K_{0}\left(\left\|x_{0}\right\|+\left\|y_{0}\right\|\right)\left\|h_{n-1}(\tau)\right\|$ a.e. on $[0, R]$. We have used the fact that $A_{n-1}(\tau)$ is dissipative (see [5]), property $\left(\mathrm{C}_{2}\right),(2.7 .8),(2.7 .9)$, and (2.7.10).

Therefore, $\left\{g_{n}\right\}$ converges uniformly to a function $g$ on $[0, R]$. Also

$$
\|g(\tau)-g(\sigma)\| \leqq\left(\left\|x_{0}\right\|+\left\|y_{0}\right\|\right)|\tau-\sigma|
$$


for $0 \leqq \sigma, \tau \leqq R$. Let $\psi(\tau)=\alpha(\tau) g(\tau), 0 \leqq \tau \leqq R$. Then

$$
\|\psi(\tau)-\psi(\sigma)\| \leqq\left(2\left\|x_{0}\right\|+\left\|y_{0}\right\|\right)|\tau-\sigma|,
$$

and $\left\|\psi(\tau)-x_{0}\right\| \leqq \delta$ for $0 \leqq \tau \leqq R$. Define the operator function $A$ on $[0, R]$ by $A(\tau)=\beta(\tau)[A(F(\tau), \psi(\tau))-I]$. Then $A$ has properties $\left(\mathrm{T}_{1}\right),\left(\mathrm{T}_{2}\right)$, and $\left(\mathrm{T}_{3}\right)$ (we will not need $\left.\left(\mathrm{T}_{3}\right)\right)$. Define $u$ on $[0, R]$ by $u(0)=x_{0}, u^{\prime}(\tau)=A(\tau) u(\tau)$.

We wish to show that $u=g$. Let $u_{n}=u-g_{n}$. An argument similar to the one used to show that $\left\{h_{n}\right\}$ converges to 0 will show that $\left\{u_{n}\right\}$ converges to 0 . Therefore $g$ satisfies $\left({ }^{* *}\right)$, and the function $f$ defined on $\left[t_{0}, c\right]$ by $f(t)=e^{t-t_{0}} g(G(t))$ satisfies $\left(^{*}\right)$. Note also that $f(t)=\psi(G(t)),\left\|f(t)-x_{0}\right\| \leqq \delta$ for $y_{0} \leqq t \leqq c$.

Suppose $x_{1} \in D_{0} \cap S, t_{0}<c_{1} \leqq c$, and $v$ is a continuously differentiable function from $\left[t_{0}, c_{1}\right]$ into $D_{0} \cap S$ such that $v\left(t_{0}\right)=x_{1}$ and $v^{\prime}(t)=A(t, v(t)) v(t)$ for $t_{0} \leqq t \leqq c_{1}$. Define $w$ on $\left[t_{0}, c_{1}\right]$ by $w(t)=f(t)-v(t)$. Then

$$
\begin{aligned}
w^{\prime}(t)= & {[A(t, f(t))-A(t, v(t))] f(t)+A(t, v(t)) w(t) } \\
= & \left([I-A(t, v(t))][I-A(t, f(t))]^{-1}-I\right)\left(f(t)-f^{\prime}(t)\right)+A(t, v(t)) w(t), \\
& (d / d t)\|w(t)\| \leqq K_{0}\|w(t)\|\left(\|f(t)\|+\left\|f^{\prime}(t)\right\|\right)
\end{aligned}
$$

a.e. on $\left[t_{0}, c_{1}\right]$. The stability claim, and hence the uniqueness claim, follow from this differential inequality.

2.8. Proof of Theorem 2. First we mention that if we prove that for each $x \in D_{0} \cap S$, there exists a continuously differentiable function $f$ from $[0, \infty)$ into $D_{0} \cap S$ such that $f(0)=x$ and $f^{\prime}(t)=Q f(t)$ for $t \geqq 0$, then the rest of the theorem follows in routine manner. We define $T_{0}(t) x=f(t)$ for $x \in D_{0} \cap S$ and $t \geqq 0$. The fact that $T_{0}$ is nonexpansive on $D_{0} \cap S$ follows from the fact that $Q$ is dissipative. Thus each $T_{0}(t)$ has a unique extension to a nonexpansive transformation $T(t)$ from $S$ into $S$. $\{T(t) ; t \geqq 0\}$ is the desired semigroup.

Now we return to the first question. Let $x_{0} \in D_{0} \cap S$. Then by Theorem 2, there is a number $c>0$ such that there is a unique continuously differentiable function $f$ from $[0, c]$ into $D_{0} \cap S$ such that $f(0)=x_{0}$ and $f^{\prime}(t)=Q f(t)$ for $0 \leqq t \leqq c$. Let $\zeta$ denote the supremum of the set of all such numbers $c$, and suppose that $\zeta<\infty$. Let $f$ denote the unique continuously differentiable function from $[0, \zeta)$ into $D_{0} \cap S$ such that $f(0)=x_{0}$ and $f^{\prime}(t)=Q f(t)$ for $0 \leqq t<\zeta$.

If $0<h<\zeta$, then define $f_{h}$ on $[0, \zeta-h]$ by $f_{h}(t)=f(t+h)-f(t)$. Then $f_{h}^{\prime}(t)=$ $Q f(t+h)-Q f(t)$, and $(d / d t)\left\|f_{h}(t)\right\| \leqq 0$ a.e. on $[0, \zeta-h)$ since $Q$ is dissipative, so that $\|(1 / h)[f(t+h)-f(t)]\| \leqq\|(1 / h)(f(h)-f(0))\|$ for $0 \leqq t<\zeta-h$. Therefore $\left\|f^{\prime}(t)\right\|$ $\leqq\left\|f^{\prime}(0)\right\|$ for $0 \leqq t \leqq \zeta$, and thus $x_{1}=\lim _{t \rightarrow \zeta} f(t)$ exists. Therefore $f([0, \zeta))$ is relatively compact and $K(t, f(t))$ (see $\left(\mathrm{C}_{2}\right)$ ) is bounded on [0, $\left.\zeta\right)$. Using this and the fact that $f(t)$ and $f^{\prime}(t)$ are also bounded on $[0, \zeta)$, we see by examining the argument for Theorem 1 that there is a positive constant $\eta$ such that for each $t$ in $[0, \zeta)$, there is a unique continuously differentiable function $g$ from $[t, t+\eta]$ into $D_{0} \cap S$ such that $g(t)=f(t)$ and $g^{\prime}(s)=Q g(s)$ for $t \leqq s \leqq t+\eta$. Simply take $\zeta-\eta<t<\zeta$, and use the corresponding function $g$ to extend $f$ beyond $\zeta$. 
3. Semigroups in a function space. Let $E$ be a set, $B(E)$ the Banach space of bounded complex valued functions on $E$ with supremum norm, and $Y$ a closed real or complex subspace of $B(E)$. We denote by $\Omega$ the collection of all positive bounded functions $p$ on $E$ which are bounded away from zero and have the property that $p Y \subset Y$.

If $Y$ is complex, we take $\mathrm{CH}(\phi)$ and $\mathrm{GH}(\phi)$ as defined in $\S 1$, with $X=Y$. If $Y$ is a real Banach lattice, then CP denotes the collection of all strongly continuous semigroups of linear positive contraction operators in $Y$, and GP denotes the collection of infinitesimal generators of such semigroups. In either case, $\mathrm{G}$ denotes the collection of all infinitesimal generators of strongly continuous semigroups of linear contraction operators in $Y$.

If $y \in Y$, then $\gamma(y)$ denotes a multiplicative linear functional on $B(E)$ such that $|\langle y, \gamma(y)\rangle|=\|y\|$. We define the semi-inner product $[\cdot, \cdot]$ on $Y \times Y$ by $[x, y]$ $=\langle x, \gamma(y)\rangle\langle y, \gamma(y)\rangle^{*}$, where ${ }^{*}$ denotes complex conjugation. All reference to a semi-inner product in this section will be to this one just defined. One special property of $[\cdot, \cdot]$ which is useful to us is that $[p x, y]=\langle p, \gamma(y)\rangle[x, y]$ for $x, y \in Y$, $p \in B(E), p x \in Y$. Also, if $Y$ is a real Banach lattice, then $[\cdot, \cdot]$ has the special properties required in [7]. That is, $[\cdot, y]$ is a positive linear functional if $y \geqq 0$, and $\left[x, x^{+}\right]=\left\|x^{+}\right\|^{2}$ for each $x$ in $Y$, where $x^{+}$denotes the positive part of $x$.

By Definition 1.2, a linear operator $A$ in $Y$ is dissipative if $\operatorname{Re}[A y, y] \leqq 0$ for $y \in D(A)$. Following [6], in case $Y$ is complex, we say that a linear operator $A$ in $Y$ is $\phi$-sectorial if $e^{i \theta} A$ is dissipative for $|\theta| \leqq \phi$. Following [7], in case $Y$ is a real lattice, we say that a linear operator $A$ in $Y$ is dispersive if $\left[A x, x^{+}\right] \leqq 0$ for all $x \in D(A)$.

Lemma 3.1. A linear operator $A$ in $Y$ is in $(\mathrm{G}, \mathrm{GP}, \mathrm{GH}(\phi))$ if and only if $D(A)$ is dense in $Y$, the range of $I-A$ is all of $Y$, and $A$ is (dissipative, dispersive, $\phi$-sectorial).

Proof. The proof of this lemma is contained in [5], [7], and [6], respectively. We merely state the lemma here for reference in proving the next theorem, which is a generalization of the author's earlier theorem in [1].

THeOrem 3.1. Suppose $A \in G$, and $A=A_{1}+\cdots+A_{n}$, where each $A_{j}$ has domain $D(A)$, and each $A_{j}$ has a closed extension. If each $A_{j}$ is (dissipative, dispersive, $\phi$-sectorial), and $p_{1}, \ldots, p_{n} \in \Omega$, then $p_{1} A_{1}+\cdots+p_{n} A_{n} \in(\mathrm{G}, \mathrm{GP}, \mathrm{GH}(\phi))$.

Proof. $p_{1} A_{1}+\cdots+p_{n} A_{n}$ is easily seen to be (dissipative, dispersive, $\phi$-sectorial). Thus by Lemma 3.1, we need only show that the range of $I-\left(p_{1} A_{1}+\cdots+p_{n} A_{n}\right)$ is all of $Y$.

We will first prove that the range of $I-\left(p_{1} A_{1}+A_{2}+\cdots+A_{n}\right)$ is all of $Y$. By [3, Lemma 2, p. 212], the operator $U_{1}=A_{1}(I-A)^{-1}$ is bounded. Since $F\left(p_{1}\right) Y \subset Y$ for every polynomial $F$, then $p_{1}^{(1 / m)} \in \Omega$ for every positive integer $m$ by the classical Weierstrass theorem. Choose $m$ so that $\left\|1-p_{1}^{(1 / m)}\right\|<\left\|U_{1}\right\|^{-1}$, and let $r=p_{1}^{1 / m}$. Then

$$
I-\left(r A_{1}+A_{2}+\cdots+A_{n}\right)=I-A+(1-r) A_{1}=\left(I+(1-r) U_{1}\right)(I-A) .
$$


Thus the range of $I-\left(r A_{1}+A_{2}+\cdots+A_{n}\right)$ is all of $Y$. Replacing $A_{1}$ by $r A_{1}, r^{2} A_{1}$, etc., we see that the range of $I-\left(p_{1} A_{1}+A_{2}+\cdots+A_{n}\right)$ is all of $Y$.

Now we consider the operator $A^{\prime}=A_{2}+p_{1} A_{1}+A_{3}+\cdots+A_{n}$ and repeat the previous argument to prove that the range of $I-\left(p_{1} A_{1}+p_{2} A_{2}+A_{3}+\cdots+A_{n}\right)$ is all of $Y$. Repeating this process proves the theorem.

ExAmplE. Let $E$ denote real Euclidean $n$-space, and let $Y$ denote any of the subspaces of $B(E)$ in which the Laplacian operator generates a strongly continuous semigroup. The semigroup will then consist of contraction operators and will be in $\mathrm{CH}(\phi)$ if $Y$ is complex, in $\mathrm{CP}$ if $Y$ is a real lattice. Let $A$ denote the Laplacian operator in $Y$, and for each $j=1, \ldots, n$, let $A_{j}$ denote the restriction of $\left(\partial^{2} / \partial s_{j}^{2}\right)$ to the domain of $A$.

Lemma 3.2. Let $A$ be in $G$ with $A=A_{1}+\cdots+A_{n}$, where each $A_{j}$ has domain $D(A)$, each $A_{j}$ has a closed extension, and each $A_{j}$ is dissipative. Define the function $P$ from $\Omega^{(n)}$ into $G$ by $P(p)=p_{1} A_{1}+\cdots+p_{n} A_{n}$.

Then there is a locally bounded nonnegative function $K$ on $\Omega^{(n)}$ such that

for $p, q \in \Omega^{(n)}$.

$$
\left\|[I-P(q)][I-P(p)]^{-1}-I\right\| \leqq\left(\sum\left\|q_{i}-p_{i}\right\|\right) K(p)
$$

Proof. If $p, q \in \Omega^{(n)}$, then

$$
[I-P(q)][1-P(p)]^{-1}-I=[P(p)-P(q)][I-P(p)]^{-1}=\sum\left(p_{i}-q_{i}\right) A_{i}[I-P(p)]^{-1} .
$$

There we can take $K(p)=\max _{i}\left\|A_{i}[I-P(p)]\right\|^{-1}$.

To see that $K(p)$ is locally bounded, notice that

$$
A_{i}(I-P(r))^{-1}=A_{i}(I-P(p))^{-1}\left(I+\sum\left(p_{i}-r_{i}\right) A_{i}(I-P(p))^{-1}\right)^{-1},
$$

so that $K(r) \leqq K(p) /\left(1-K(p) \sum\left\|p_{i}-r_{i}\right\|\right)$ for

$$
K(p) \sum\left\|p_{i}-r_{i}\right\|<1 .
$$

THEOREM 3.3. Let $B$ be in $\mathrm{GH}(\phi)$ with $B=B_{1}+\cdots+B_{n}$, where each $B_{j}$ has a closed extension, each $B_{j}$ has domain $D(A)=D_{0}$, and each $B_{j}$ is $\phi$-sectorial. Let $S$ be a closed set in $Y,[a, b]$ a closed interval, and $p$ a Lipschitz continuous function from $[a, b] \times S$ into $\Omega^{(n)}$. Define the operator function $A$ from $[a, b] \times S$ into $\mathrm{GH}(\phi)$ by $A(t, x)=\sum p_{i}(t, x) A_{i}$. Then $A$ satisfies conditions $\left(\mathrm{C}_{1}\right)$ and $\left(\mathrm{C}_{2}\right)$.

Proof. This follows from Lemma 3.2.

There are a variety of ways in which the set $S$ in Theorem 3.2 could be chosen in order that the operator function $A$ will satisfy $\left(C_{3}\right)$, and it seems inappropriate to state any theorems about this. It is not quite so easy to choose $H$ and $S$ so that $Q$ will be dissipative as in Theorem 2, but we will indicate one way in which it can be done.

Let $Y$ be complex, let $Y_{0}$ denote the space of real functions in $Y$, and suppose $Y_{0}$ is a lattice. Let $A \in \mathrm{GH}(\phi), A=A_{1}+\cdots+A_{n}$ as in Theorem 3.2. Let $D_{00}=D_{0} \cap Y_{0}$, 
$A^{0}=\left.A\right|_{D_{00}}, A_{j}^{0}=\left.A_{j}\right|_{D_{00}}$, and suppose that $A^{0}, A_{j}^{0}$ satisfy the portion of Theorem 3.1 dealing with positive semigroups. Let $Y_{00}$ denote the nonpositive functions in $Y_{0}$, let $S_{0}=\bigcap\left(A_{j}^{0}\right)^{-1} Y_{00}$, and let $S$ denote the closure of $S_{0}$. Let $p_{1}, \ldots, p_{n}$ be Lipschitz continuous accretive $\left(-p_{j}\right.$ dissipative) functions from $S$ into $\Omega$. Define $H$ from $S$ onto $\mathrm{GH}(\phi)$ by $H(x)=\sum p_{i}(x) A_{i}$. Then the hypothesis of Theorem 2 is satisfied. This can all be done taking $Y, A, A_{j}$ as in the example after Theorem 3.1 which dealt with the Laplacian operator.

\section{REFERENCES}

1. J. R. Dorroh, Contraction semi-groups in a function space, Pacific J. Math. 19 (1966), 35-38. MR 34 \#1860.

2. E. Hille and R. S. Phillips, Functional analysis and semi-groups, rev. ed., Amer. Math. Soc. Colloq. Publ., vol. 31, Amer. Math. Soc., Providence, R. I., 1957. MR 19, 664.

3. T. Kato, Integration of the equation of evolution in a Banach space, J. Math. Soc. Japan 5 (1953), 208-234. MR 15, 437.

4. - Nonlinear semigroups and evolution equations, J. Math. Soc. Japan 19 (1967), 508-520. MR $37 \# 1820$.

5. G. Lumer and R. S. Phillips, Dissipative operators in a Banach space, Pacific J. Math. 11 (1961), 679-698. MR 24 \#A2248.

6. R. T. Moore, Duality methods and perturbation of semigroups, Bull. Amer. Math. Soc. 73 (1967), 548-553. MR 36 \#5759.

7. R. S. Phillips, Semi-groups of positive contraction operators, Czechoslovak Math. J. 12 (87) (1962), 294-313. MR 26 \#4195.

8. H. Tanabe, On the equations of evolution in a Banach space, Osaka Math. J. 12 (1960), 363-376. MR 23 \#A2756b.

Louisiana State University, Baton Rouge, Louisiana 